Cite this: J. Mater. Chem. C, 2014, 2, 2346

Received 15th November 2013 Accepted 9th January 2014

DOI: $10.1039 / c 3 t c 32260 b$

www.rsc.org/MaterialsC

\section{Thermoelectric properties of a single graphene sheet and its derivatives $\uparrow$}

\begin{abstract}
A. H. Reshak, ${ }^{\text {ab }}$ Saleem Ayaz Khan ${ }^{\star a}$ and S. Auluck ${ }^{c}$
The thermoelectric properties of pristine graphene and $\mathrm{H}_{2} \mathrm{~S}$ adsorbed onto bridge, hollow and top sites of a graphene sheet are investigated using the semi-classical Boltzmann transport theory. The average values of electrical conductivity, thermal conductivity, Seebeck coefficient, figure of merit $(Z T)$ and the average value of the power factor $\left(P^{\mathrm{av}}\right)$ are reported and discussed in detail. While pristine graphene is a zero band gap semiconductor, adsorption of $\mathrm{H}_{2} \mathrm{~S}$ onto the bridge site opens up a direct energy gap of about $0.04 \mathrm{eV}$, adsorption of a $\mathrm{H}_{2} \mathrm{~S}$ molecule onto the top site opens up a gap of $0.3 \mathrm{eV}$, and adsorption of $\mathrm{H}_{2} \mathrm{~S}$ onto the hollow site makes it metallic. The investigation of $Z T$ and power factor values suggests that a top-site configuration could be a potential candidate for thermoelectric applications in the range 300-600 K.
\end{abstract}

\section{Introduction}

The increasing energy demand is a global problem that needs to be addressed. On the other hand the combustion of fossil fuels affects the climate causing global warming. ${ }^{1}$ Thermoelectric power generators can convert low grade heat into electricity and appear to be a good solution because of their environmentally friendly behavior and reasonable cost. ${ }^{2}$ Extensive work has been done on different materials in order to investigate and enhance the thermoelectric properties for technological applications. ${ }^{3-12}$ Hence searching for novel thermoelectric materials is interesting for both theoreticians and experimentalists.

Graphene is a honeycomb two-dimensional lattice of carbon atoms. As a result of its many interesting properties graphene seems to be a rapidly rising star on the horizon of materials science, engineering and technology. Graphene exhibits exceptionally high electronic properties, high efficiency, low cost and higher strength to weight ratios. In recent past, it has already revealed a copiousness of new physics and potential applications in ultracapacitors, integrated circuits, hydrogen storage materials, transparent conducting electrodes, sensors and biodevices. ${ }^{13-17}$ It has unique thermal and thermoelectric properties. ${ }^{18,19}$ Graphene exhibits excellent electrical conductivity $^{20}$ however its exceedingly high thermal conductivity makes it a poor contestant for thermoelectric applications. ${ }^{21} \mathrm{On}$

${ }^{a}$ New Technologies - Research Centre, University of West Bohemia, Univerzitni 8, 306 14 Pilsen, Czech republic. E-mail: sayaz_usb@yahoo.com; maalidph@yahoo.co.uk; Tel: +420777729583

${ }^{b}$ Center of Excellence Geopolymer and Green Technology, School of Material Engineering, University Malaysia Perlis, 01007 Kangar, Perlis, Malaysia

${ }^{c}$ Council of Scientific and Industrial Research - National Physical Laboratory, Dr K S, Krishnan Marg, New Delhi 110012, India

$\dagger$ Electronic supplementary information (ESI) available. See DOI: $10.1039 / \mathrm{c} 3 \mathrm{tc} 32260 \mathrm{~b}$ the other hand, different modifications can be applied to the structure of graphene in order to reduce its thermal conductivity and thus increase the figure of merit. Pristine graphene is chemically inert because of strong $\mathrm{sp}^{2}$ binding between carbon atoms in the graphene plane. Extensive work has been done by experimentalists and theoreticians in order to understand and improve the physical and chemical properties of graphene nanosheets by means of adsorption of different chemical species $\left(\mathrm{CO}, \mathrm{N}_{2} \mathrm{O}, \mathrm{NH}_{3}\right.$ and $\mathrm{CO}_{2}$ ) on its surface. ${ }^{22-25}$ The DNAdecorated graphene elucidates high efficiency as chemical sensors. $^{22}$ Moreover multilayer graphene nanoribbons $(\text { MLGNR })^{26}$ personalized with Pd can be used as active materials for sensors and hydrogen storage materials. ${ }^{27,28}$ The previous literature exposed that organic electron donors, ${ }^{29}$ acceptors ${ }^{30}$ or even DNA fragments ${ }^{31}$ can induce considerable changes in the electronic structure of graphene which make it suitable for new devices with optimized performances. $\mathrm{H}_{2} \mathrm{~S}$ is a foul-smelling and poisonous gas which is imperative in environmental observation. Therefore much attention has been given to it. At higher concentration (more than $250 \mathrm{ppm}$ ) it can cause death. ${ }^{32}$ The previous work ${ }^{\mathbf{3}, 34}$ exposed that pristine graphene is a good absorbent of $\mathrm{H}_{2} \mathrm{~S}$ on a single graphene nanosheet and can be used as a promising contestant for detection of a single molecule of $\mathrm{H}_{2} \mathrm{~S}$.

Although many properties of pristine graphene have been studied by many researchers, there is not much work on the transport properties. Hence in the present work we report the investigation of the transport properties such as conductivity and Seebeck coefficient of pristine graphene. We also explore the effect of depositing $\mathrm{H}_{2} \mathrm{~S}$ on a pristine graphene sheet at different sites with a view to ascertain if such materials could be good thermoelectric devices. We estimate the figure of merit $(Z T)$, a dimensionless quantity that characterizes the thermoelectric properties. ${ }^{35}$ 


\section{Computational details}

Pristine graphene single sheet is a zero gap semiconductor with exceptional properties. To enhance and/or modify these properties a single $\mathrm{H}_{2} \mathrm{~S}$ molecule is adsorbed at different sites namely; the top site on the top of the carbon atom, the bridge site in the middle of the $\mathrm{C}-\mathrm{C}$ bond, and the hollow site at the center of the carbon hexagon. The BoltzTraP program was employed to investigate the thermoelectric properties based on the analytical expressions of the electronic bands. This program calculates semiclassical transport based on the all electron full potential linear augmented plane wave (FPLAPW) method within the framework of the WIEN2K code. ${ }^{36}$ The constant relaxation time approximation and the rigid band approximation were used in the calculations. ${ }^{37}$ The self consistent calculation was done using Perdew-Burke-Ernzerh of generalized gradient approximation (GGA-PBE).$^{38}$ In order to converge the energy eigenvalues the wave functions in the interstitial regions were expanded in plane waves with cutoff $R_{\mathrm{MT}} K_{\max }=7.0$. Here $R_{\mathrm{MT}}$ represents the muffin-tin sphere radius and $K_{\max }$ corresponds to the magnitude of the largest $K$ vector in plane wave expansion. The selected $R_{\mathrm{MT}}$ is 1.33 a.u. for $\mathrm{C}$ in a $4 \times 4$ graphene sheet (32 $\mathrm{C}$ atoms) and 1.27, 1.55 and 0.83 a.u. for $\mathrm{C}, \mathrm{S}$, and $\mathrm{H}$ atoms, respectively at top, bridge, and hollow sites of $\mathrm{H}_{2} \mathrm{~S}$ adsorption onto graphene. The wave function inside the sphere was expanded up to $l_{\max }=10$ whereas the Fourier expansion of the charge density was up to $G_{\max }=20$ (a.u.) ${ }^{-1}$. For investigating the thermoelectric properties a denser mesh of $2500 \mathrm{k}$ points in the IBZ was used. The self-consistent calculations are considered to be converged when the total energy of the system is stable within $10^{-5} \mathrm{Ry}$.

\section{Results and discussions}

\subsection{Salient features of the electronic band structures and thermoelectric properties}

Let us recall the main features of the electronic band structure of pristine graphene sheet, bridge, top, and hollow configurations. The pristine graphene sheet is a zero gap semiconductor, the bridge configuration has a small energy gap of about 0.04 $\mathrm{eV}$, the top configuration shows a bigger direct energy band gap of about $0.3 \mathrm{eV}$, while the hollow configuration is metallic. We should emphasize that the most stable adsorption configuration is the one with the lowest total energy among other adsorption sites. ${ }^{39}$ Thus the top site (total energy $\left(E_{\mathrm{TOT}}\right)=$ $-3238.87878541 \mathrm{Ry})$ is the most stable configuration in comparison to (hollow $E_{\mathrm{TOT}}=-3238.1058284$ Ry and bridge $E_{\mathrm{TOT}}=-3238.79501144$ Ry in comparison to pristine graphene $\left.E_{\mathrm{TOT}}=-2438.50922329 \mathrm{Ry}\right)$.

As there is no experimental work done on the thermoelectric properties of $\mathrm{H}_{2} \mathrm{~S}$ adsorbed graphene therefore we do not have any information about the structure stability of graphene derivatives (bridge, top and hollow). Zhang et al. ${ }^{40}$ proved that the oxygen molecule adsorbed graphene/Ru (0001) surface shows high stability at $600 \mathrm{~K}$ and when the temperature increased to $800 \mathrm{~K}$ the surface oxygen is removed. Therefore we investigated the thermoelectric properties of pristine graphene and its derivatives in the temperature range between $100 \mathrm{~K}$ and $600 \mathrm{~K}$ and avoided the higher temperature in order to maintain the structure stability of $\mathrm{H}_{2} \mathrm{~S}$ on the graphene nanosheet. However we have done calculation up to $800 \mathrm{~K}$ and we find that the power factor becomes maximum at around $600 \mathrm{~K}$. The calculated spectra of carrier concentration vs. chemical potential for the four configurations (pristine graphene, bridge, top and hollow) at $300 \mathrm{~K}$ and $600 \mathrm{~K}$, respectively are shown in Fig. 1a-d along with the electronic band structure in the energy region between 2.0 and $-2.0 \mathrm{eV}$. Also shown in the insets are the various structures used in our calculations. For the case of bridge configuration (Fig. 1b), the band structure shows a gap of $0.2 \mathrm{eV}$ at symmetry point $K$, while the minimum gap of $0.04 \mathrm{eV}$ (as revealed by the density of states, which are not presented here) occurs at a nonsymmetry point. The difference between chemical potential and Fermi energy $\left(\mu-\varepsilon_{\mathrm{f}}\right)$ is positive for the valence band and negative for the conduction band. In the case of pristine graphene the carrier concentration shows a zero value at the Fermi level and there is no significant difference in carrier concentration at two different temperatures $(300 \mathrm{~K}$ and $600 \mathrm{~K})$. The bridge-site configuration shows zero carrier concentration in the narrow band gap region at room temperature $(300 \mathrm{~K})$, while at $600 \mathrm{~K}$ there is a negligible increase in carrier concentration. The topsite configuration shows zero electron concentration in a small region at $300 \mathrm{~K}$. The width of the curve decreases for maximum temperature $(600 \mathrm{~K})$ suggesting that there is a small increase in electron concentration while the hole concentration is almost the same. In the case of the hollow-site the valence band maximum and conduction band minimum strongly overlap around the Fermi level resulting in a maximum carrier concentration around the Fermi level.

Thermoelectric transport tensors can be evaluated on the basis of the calculated band structure using the standard Boltzmann kinetic transport theory and the rigid band approach. ${ }^{37}$ The electrical conductivity $\sigma_{\alpha \beta}$, Seebeck coefficient $S_{\alpha \beta}$ and thermal conductivity (electronic part) $k_{\alpha \beta}^{0}$ tensors are the main transport properties. These quantities are functions of temperature $(T)$ and chemical potential $(\mu)::^{37,41}$

$$
\begin{gathered}
\sigma_{\alpha \beta}(T ; \mu)=\frac{1}{\Omega} \int \sigma_{\alpha \beta}(\varepsilon)\left[-\frac{\partial \mathrm{f}_{\mu}(T ; \varepsilon)}{\partial \varepsilon}\right] \mathrm{d} \varepsilon \\
S_{\alpha \beta}(T ; \mu)=\frac{1}{e T \Omega \sigma_{\alpha \beta}(T ; \mu)} \int \sigma_{\alpha \beta}(\varepsilon)(\varepsilon-\mu) \times\left[-\frac{\partial \mathrm{f}_{\mu}(T ; \varepsilon)}{\partial \varepsilon}\right] \mathrm{d} \varepsilon \\
k_{\alpha \beta}^{0}(T ; \mu)=\frac{1}{e^{2} T} \int \sigma_{\alpha \beta}(\varepsilon)(\varepsilon-\mu)^{2}\left\{-\frac{\partial \mathrm{f}_{\mu}(T ; \varepsilon)}{\partial \varepsilon}\right\} \mathrm{d} \varepsilon
\end{gathered}
$$

where

$$
\sigma_{\alpha \beta}(\varepsilon)=\frac{e^{2}}{N} \sum_{i, k} \tau_{i}, k v_{\alpha}(i, k) v_{\beta}(i, k) \delta\left(\varepsilon-\varepsilon_{i, k}\right)
$$

where $\alpha$ and $\beta$ symbolize the tensor indices, $e$ represents the charge of the electron, $\Omega$ stands for the volume of the unit cell, $N$ is the number of $k$-points sampled, $\varepsilon(k)$ is the band energy, $v(k)$ represents the band velocity, $\tau(k)$ is the relaxation time and $f$ is the Fermi function. The transport properties of the investigated 


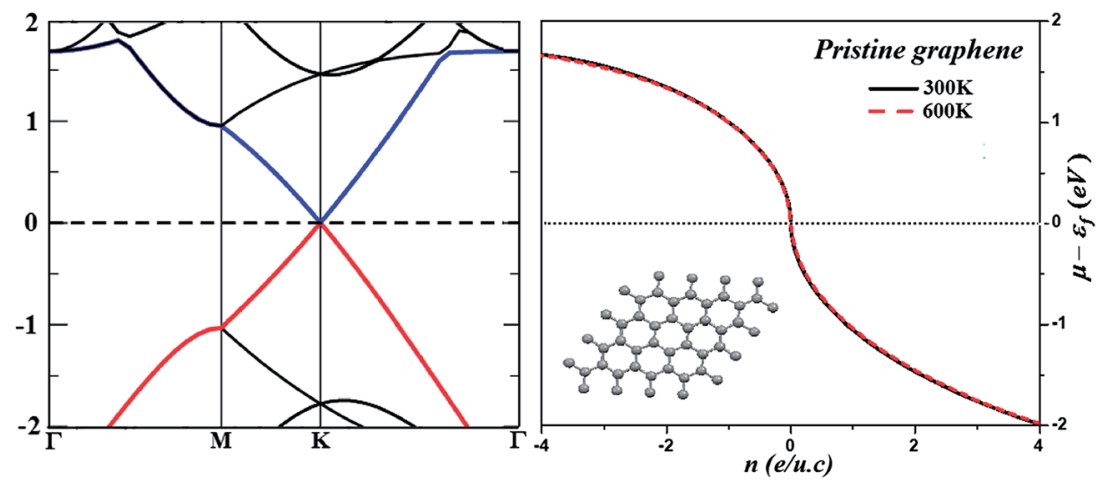

(a)
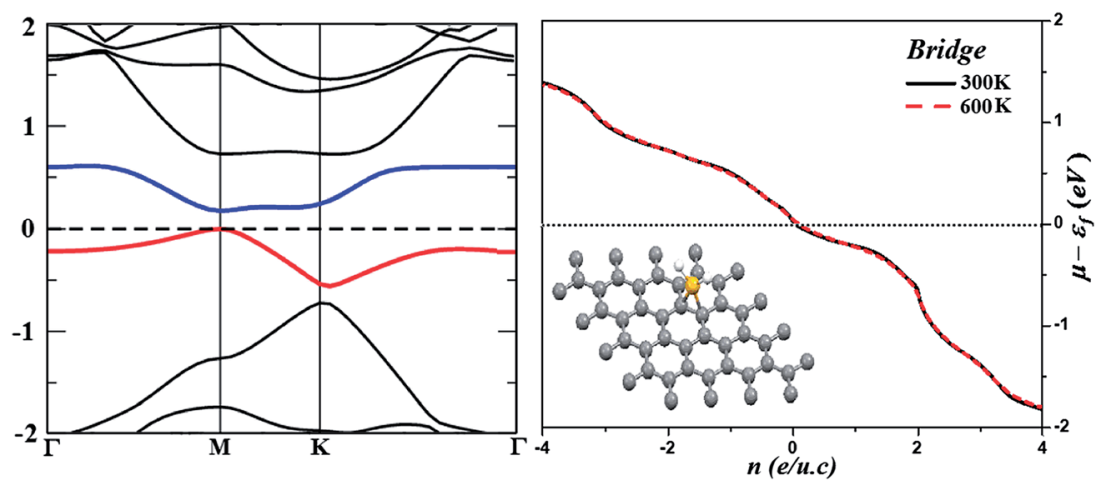

(b)

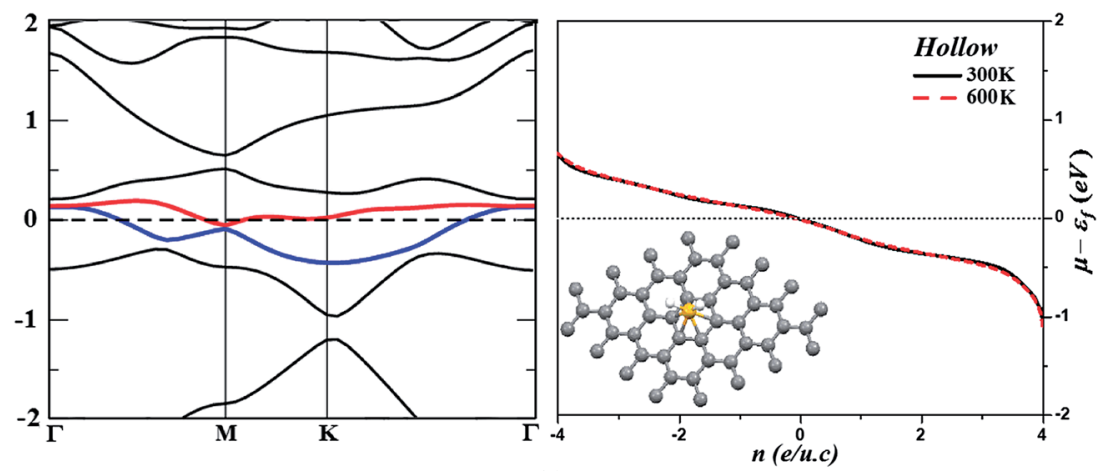

(c)
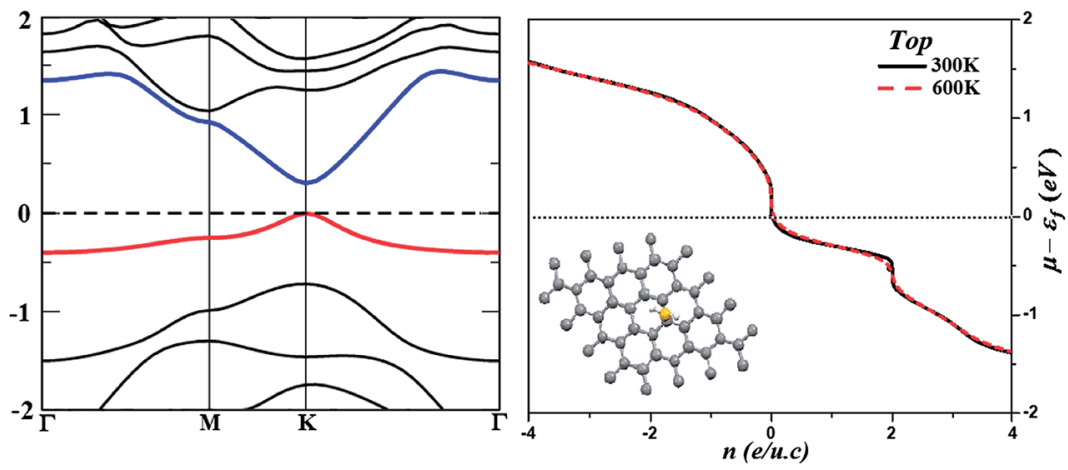

(d)

Fig. 1 Calculated band structure and carrier concentration of (a) pristine graphene, (b) $\mathrm{H}_{2} \mathrm{~S}$ adsorption on the bridge site, (c) $\mathrm{H}_{2} \mathrm{~S}$ adsorption on the hollow site and (d) $\mathrm{H}_{2} \mathrm{~S}$ adsorption on the top site.

compounds were calculated using the BoltzTrap program ${ }^{37}$ which depends on a well tested smoothed Fourier interpolation to obtain an analytical expression of bands. This is based on the fact that the electrons contributing to transport are in a narrow energy range due to the delta-function like Fermi broadening. For such a narrow energy range the relaxation time is nearly the 
same for the electrons. The accuracy of this method has been tested earlier, and the method actually turns out to be a good approximation. ${ }^{42}$ The temperature dependence of the energy band structure is ignored. It is suggested below that the high thermoelectric efficiency of the material is due to high electrical conductivity, large Seebeck coefficient and low thermal conductivity. ${ }^{43}$

The crystal structures of pristine graphene, bridge site, hollow site and top site configurations are stable in hexagonal symmetry which contains two non-zero diagonal components of the second order dielectric tensor along $a, b$ and $c$ crystallographic axes. For simplicity we calculated the average values of dominant tensor components for electrical conductivity, Seebeck coefficient, thermal conductivity and power factor, respectively.

3.1.1. Electrical conductivity. Fig. 2 shows the average values of the electrical conductivity tensor component $\left(\sigma^{\text {av }}\right)$ plot for pristine graphene, bridge, top and hollow site configurations. The relaxation time $\tau$ is taken to be direction independent and isotropic. ${ }^{44}$ The $\tau$ value $\left(3.0 \times 10^{-13} \mathrm{~s}\right)$ is taken from ref. 45 . For pristine graphene $\left(\sigma^{\mathrm{av}}\right)$ smoothly increases with temperature and shows a maximum value around $3.0 \times 10^{6}(\Omega \mathrm{m})^{-1}$ at $600 \mathrm{~K}$ whereas the bridge-site elucidates a maximum value of about $1.9 \times 10^{6}(\Omega \mathrm{m})^{-1}$ around $100 \mathrm{~K}$ which gradually increases with temperature and shows a maximum value of $7.0 \times 10^{6}(\Omega$ $\mathrm{m})^{-1}$ and $12.0 \times 10^{6}(\Omega \mathrm{m})^{-1}$ at $300 \mathrm{~K}$ and $600 \mathrm{~K}$. The top-site shows a minimum value of $0.8 \times 10^{6}(\Omega \mathrm{m})^{-1}$ at $100 \mathrm{~K}$, as we move towards higher temperatures there is a linear increase in the conductivity value. Finally, the hollow-site shows a maximum value of about $37.6 \times 10^{6}(\Omega \mathrm{m})^{-1}$ at $100 \mathrm{~K}$. For higher temperature $(600 \mathrm{~K})$ the spectra shift to $31.2 \times 10^{6}(\Omega \mathrm{m})^{-1}$. The pristine graphene is a zero band gap semiconductor, bridge and top-site configurations are narrow band gap semiconductors while the hollow site shows metallic behavior. For the first three cases (pristine graphene, bridge and top-site configurations) the electron transition to the conduction band occurs even at lower temperature to some extent. Further increase in the

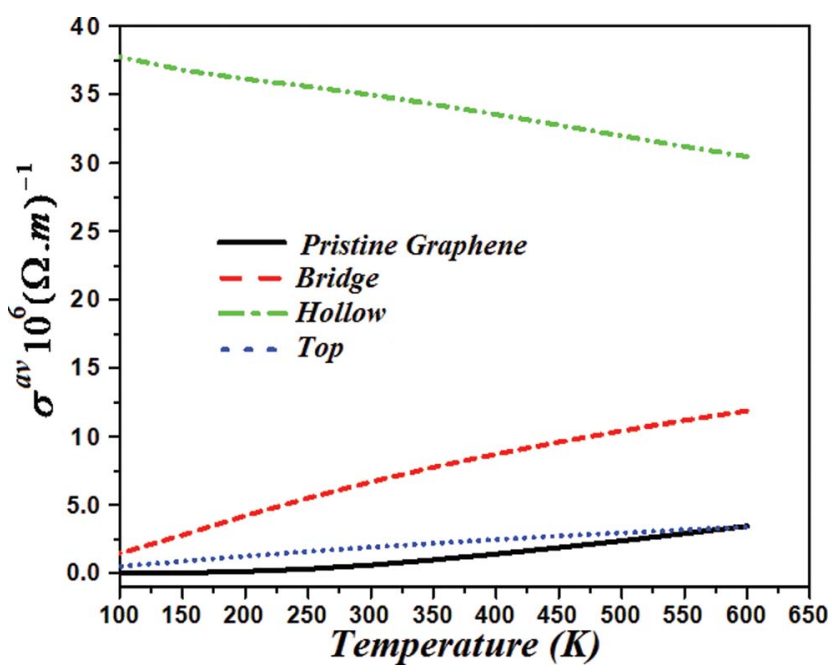

Fig. 2 Calculated average electrical conductivity ( $\sigma^{\mathrm{av}}$ ) of pristine graphene, $\mathrm{H}_{2} \mathrm{~S}$ adsorption on the bridge site, $\mathrm{H}_{2} \mathrm{~S}$ adsorption on the hollow site and $\mathrm{H}_{2} \mathrm{~S}$ adsorption on the top site. temperature results to enhance the electron thermal energy which enables the electrons to rise above the energy barrier (bandgap) to the conduction band and thus the conductivity of pristine graphene, bridge- and top-site configurations increases with increasing temperature. For hollow-site configuration the situation is different, the flow of electrons can occur easily because of its metallic behavior. When temperature is increased the collision rate of conduction electrons with lattice phonons is increased which causes a decrease in the electrical conductivity (due to the scattering electrons) ${ }^{46}$ as shown in Fig. 2.

3.1.2. Seebeck coefficient. Seebeck coefficient is the main thermoelectric transport property. Actually it decides the efficiency of thermocouples. It is related to the fact that electrons carry both heat and charge. The diffusion of the electron depends on temperature gradient present in the material which creates the opposite electric field $(E)$ and hence voltage known as Seebeck voltage. The trend of the spectra in the Seebeck coefficient and electronic conductivity depends on the Seebeck voltage. Its magnitude and sign are correlated with an asymmetry distribution of electrons around the Fermi level. ${ }^{47}$ The asymmetric energy distribution of electrons moving in the material related to the Fermi energy gives a greater value of Seebeck coefficient. On the other hand reduction in Joule heating results in a decrease in conductivity. The average value of Seebeck coefficient ( $S^{\text {av }}$ ) shows a maximum of about $223 \mu \mathrm{V}$ $\mathrm{K}^{-1}$ for pristine graphene at $100 \mathrm{~K}$ (Fig. 3). There is a significant decrease in $S^{\text {av }}$ up to $300 \mathrm{~K}\left(31 \mu \mathrm{V} \mathrm{K} \mathrm{K}^{-1}\right)$. Further increasing the temperature above $300 \mathrm{~K}$ results in a smooth decrease in the curve which is finally reduced to $20 \mu \mathrm{V} \mathrm{K}^{-1}$ at $600 \mathrm{~K}$. The bridgesite configuration Seebeck coefficient has a value around $71.5 \mu \mathrm{V} \mathrm{K}^{-1}$ at $100 \mathrm{~K}$. It reduced to $59 \mu \mathrm{V} \mathrm{K}^{-1}$ at $400 \mathrm{~K}$ and then the curve became smooth towards higher energy with a minor shift of $2.0 \mu \mathrm{V} \mathrm{K}^{-1}$. The hollow-site configuration shows a small value of $S^{\text {av }}\left(8.0 \mu \mathrm{V} \mathrm{K}^{-1}\right)$ as compared to the other three cases at $100 \mathrm{~K}$. For higher temperature $(600 \mathrm{~K})$ the $S^{\text {av }}$ increased to $14 \mu \mathrm{V}$ $\mathrm{K}^{-1}$. The top-site gives higher values for $S^{\mathrm{av}}$ as compared to the

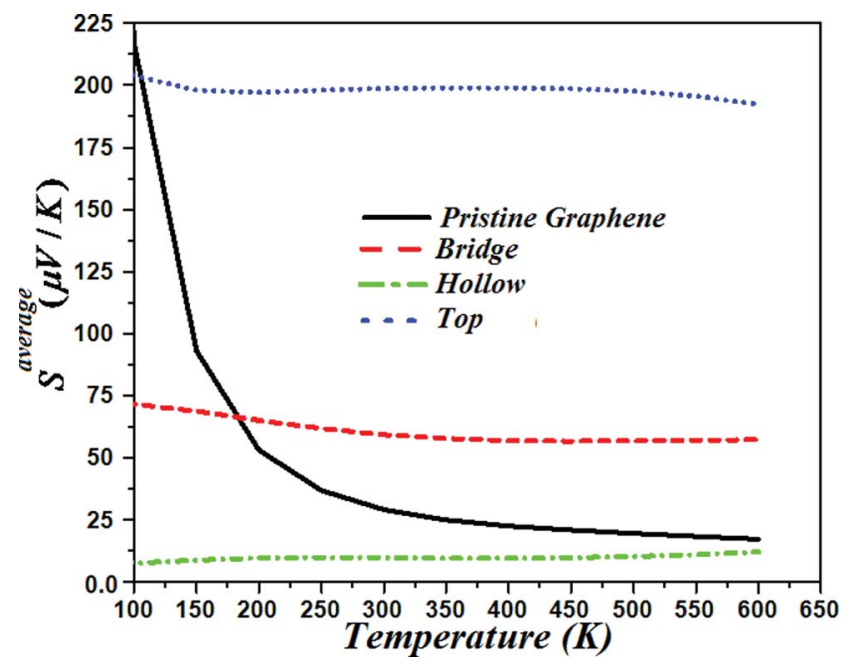

Fig. 3 Calculated average Seebeck coefficient $\left(S^{\text {av }}\right)$ of pristine graphene, $\mathrm{H}_{2} \mathrm{~S}$ adsorption on the bridge site, $\mathrm{H}_{2} \mathrm{~S}$ adsorption on the hollow site and $\mathrm{H}_{2} \mathrm{~S}$ adsorption on the top site. 
other three cases. At $100 \mathrm{~K} \mathrm{~S}^{\mathrm{av}}$ is $223 \mu \mathrm{V} \mathrm{K} \mathrm{K}^{-1}$. It decreased to $197 \mu \mathrm{V} \mathrm{K}^{-1}$ around $200 \mathrm{~K}$ which again increased to $200 \mu \mathrm{V} \mathrm{K}^{-1}$ at $400 \mathrm{~K}$. The spectra of $S^{\mathrm{av}}$ reduced to $193.5 \mu \mathrm{V} \mathrm{K} \mathrm{K}^{-1}$ at $600 \mathrm{~K}$.

3.1.3. Thermal conductivity. Thermal conductivity is physical quantity of a material that conducts heat. The overall trend of thermal conductivity spectra shows that more transfer of heat takes place as one moves towards higher temperatures which results in a linear increase in thermal conductivity with temperature. In real systems the behavior is more complicated. The thermal conductivity has contributions from the lattice and electrons. BoltzTraP calculates the electronic part. The average electronic thermal conductivity $\left(k^{\text {av }}\right)$ of pristine graphene is nearly zero at $100 \mathrm{~K}$ as illustrated in Fig. 4. One can see that there is a negligible increase in the thermal conductivity up to $200 \mathrm{~K}$, after that the thermal conductivity increases rapidly with

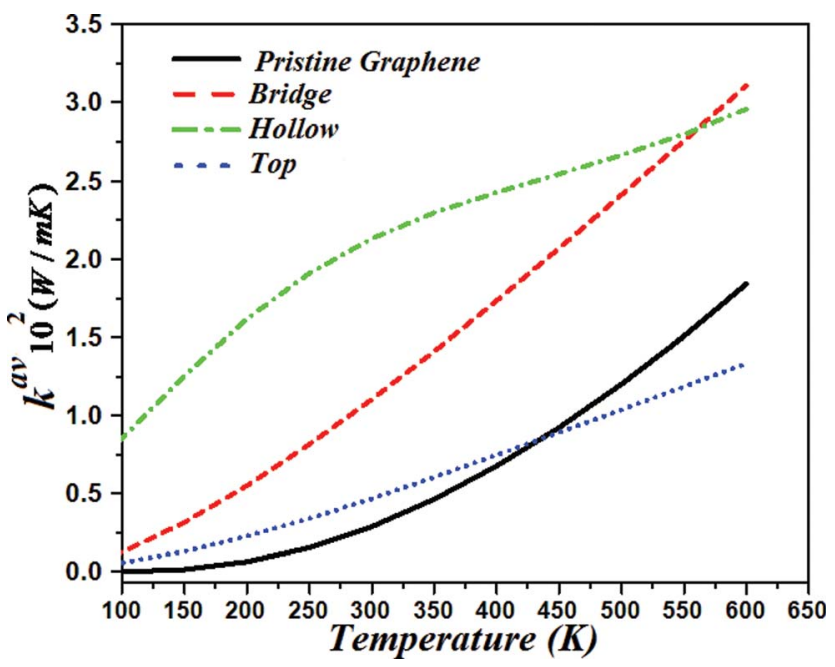

Fig. 4 Calculated average thermal conductivity ( $k^{\mathrm{av}}$ ) of pristine graphene, $\mathrm{H}_{2} \mathrm{~S}$ adsorption on the bridge site, $\mathrm{H}_{2} \mathrm{~S}$ adsorption on the hollow site and $\mathrm{H}_{2} \mathrm{~S}$ adsorption on the top site. the temperatures. The maximum value of thermal conductivity is $1.76 \times 10^{2} \mathrm{~W} \mathrm{~m}^{-1} \mathrm{~K}^{-1}$ at $600 \mathrm{~K}$. The bridge sites have the value of $k^{\text {av }}$ equal to $0.15 \times 10^{2} \mathrm{~W} \mathrm{~m}^{-1} \mathrm{~K}^{-1}$ at $100 \mathrm{~K}$, which gradually increased to $3.1 \times 10^{2} \mathrm{~W} \mathrm{~m}^{-1} \mathrm{~K}^{-1}$ at $600 \mathrm{~K}$. The hollow site configuration had a greater value of $k^{\mathrm{av}}\left(0.81 \times 10^{2} \mathrm{~W} \mathrm{~m}^{-1} \mathrm{~K}^{-1}\right)$ at $100 \mathrm{~K}$ which further increased to $2.125 \times 10^{2} \mathrm{~W} \mathrm{~m}{ }^{-1} \mathrm{~K}^{-1}$, $2.43 \times 10^{2} \mathrm{~W} \mathrm{~m}^{-1} \mathrm{~K}^{-1}$ and $2.94 \times 10^{2} \mathrm{~W} \mathrm{~m}^{-1} \mathrm{~K}^{-1}$ at $300 \mathrm{~K}, 400 \mathrm{~K}$ and $600 \mathrm{~K}$, respectively. The top-site shows zero conductivity at $100 \mathrm{~K}$ which linearly increases showing a maximum value of about $1.26 \times 10^{2} \mathrm{~W} \mathrm{~m}^{-1} \mathrm{~K}^{-1}$ at $600 \mathrm{~K}$.

3.1.4. Figure of merit. The thermoelectric performance of a material is characterized by the dimensionless figure of merit $(Z T)$. In the expression $Z T=S^{2} T \sigma /\left(k_{\mathrm{el}}+k_{\mathrm{ph}}\right)^{48}$ where $k_{\mathrm{el}}$ and $k_{\mathrm{ph}}$ are the thermal conductivities attributed to electrons and phonons (lattice), respectively. The phonon thermal conductivities of the lattice for pristine graphene for the temperatures $200,300,400$ and $500 \mathrm{~K}$ are taken from ref. 48 . As there is no experimental value of phonon thermal conductivities for bridge, hollow and top site configurations, we take the phonon thermal conductivity for these cases to be the same as that of pristine graphene. The total thermal conductivity and the calculated values of $Z T$ for pristine graphene and $\mathrm{H}_{2} \mathrm{~S}$ on graphene for different temperatures are reported in Table 1 . The table suggests that a high performance of the top-site configuration may make it suitable for thermoelectric applications.

3.1.5. Power factor. Power factor is also a keynote quantity for investigating transport properties. The calculated values of power factor $\left(P^{\mathrm{av}}=S^{2} \sigma\right)$ for pristine graphene, bridge, hollow and top sites are shown in Fig. 5. Pristine graphene shows a nearly zero value for the whole range of temperatures. The bridge-site gives a $P^{\text {av }}$ of $0.75 \times 10^{-3} \mathrm{~W} \mathrm{~m}^{-1} \mathrm{~K}^{-2}$ at $100 \mathrm{~K}$. This configuration attains the value of $2.3 \times 10^{-3} \mathrm{~W} \mathrm{~m}^{-1} \mathrm{~K}^{-2}$ at room temperature $(300 \mathrm{~K})$. Further increasing the temperature results in a linear increase in the spectra and it finally shows a maximum value of $3.5 \times 10^{-3} \mathrm{~W} \mathrm{~m}^{-1} \mathrm{~K}^{-2}$ at $600 \mathrm{~K}$. While in the case of the hollowsite the $P^{\mathrm{av}}$ shows a small value of $0.3 \times 10^{-3} \mathrm{~W} \mathrm{~m}^{-1} \mathrm{~K}^{-2}$ at $100 \mathrm{~K}$.

Table 1 Calculated value thermal conductivity $K / \tau\left(\mathrm{W} \mathrm{m} \mathrm{m}^{-1} \mathrm{~K}^{-1} \mathrm{~s}^{-1}\right)$ and figure of merit $(Z T)$ for pristine graphene with relaxation time $\tau=3.0 \times 10^{-13} \mathrm{~s}$

\begin{tabular}{|c|c|c|c|c|c|}
\hline & Temp (K) & Lattice $K / \tau$ & Electronic $K / \tau^{a}$ & Total $K / \tau^{a}$ & $Z T^{a}$ \\
\hline \multirow[t]{3}{*}{ Pristine graphene } & 200 & $0.25 \times 10^{14 b}$ & $0.30 \times 10^{14}$ & $0.55 \times 10^{14}$ & $1.9 \times 10^{-2}$ \\
\hline & 300 & $0.47 \times 10^{14 b}$ & $0.85 \times 10^{14}$ & $1.32 \times 10^{14}$ & $8.0 \times 10^{-2}$ \\
\hline & 500 & $0.78 \times 10^{14 b}$ & $4.13 \times 10^{14}$ & $4.91 \times 10^{14}$ & $4.0 \times 10^{-2}$ \\
\hline \multirow[t]{2}{*}{ Bridge-site } & 200 & $0.25 \times 10^{14 b}$ & $2.00 \times 10^{14}$ & $2.25 \times 10^{14}$ & $5.4 \times 10^{-2}$ \\
\hline & 300 & $0.47 \times 10^{14 b}$ & $3.75 \times 10^{14}$ & $4.22 \times 10^{14}$ & $6.9 \times 10^{-2}$ \\
\hline \multirow[t]{4}{*}{ Hollow-site } & 200 & $0.25 \times 10^{14 b}$ & $5.50 \times 10^{14}$ & $5.75 \times 10^{14}$ & $4.3 \times 10^{-3}$ \\
\hline & 300 & $0.47 \times 10^{14 b}$ & $7.06 \times 10^{14}$ & $7.53 \times 10^{14}$ & $7.2 \times 10^{-3}$ \\
\hline & 400 & $0.68 \times 10^{14 b}$ & $7.91 \times 10^{14}$ & $8.59 \times 10^{14}$ & $7.8 \times 10^{-3}$ \\
\hline & 500 & $0.78 \times 10^{14 b}$ & $8.73 \times 10^{14}$ & $9.51 \times 10^{14}$ & $8.8 \times 10^{-3}$ \\
\hline \multirow[t]{2}{*}{ Top-site } & 200 & $0.25 \times 10^{14 b}$ & $0.37 \times 10^{14}$ & $0.63 \times 10^{14}$ & 0.51 \\
\hline & 300 & $0.47 \times 10^{14 b}$ & $0.83 \times 10^{14}$ & $1.30 \times 10^{14}$ & 0.56 \\
\hline
\end{tabular}

${ }^{a}$ Present work. ${ }^{b}$ Ref. 48. 


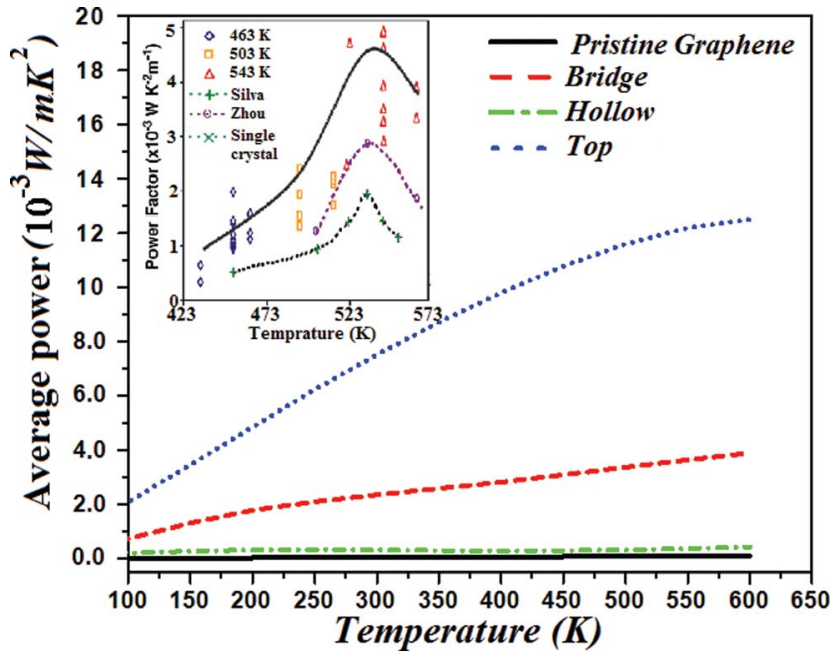

Fig. 5 Calculated average value of power factor $\left(P^{\text {av }}\right)$ of pristine graphene, $\mathrm{H}_{2} \mathrm{~S}$ adsorption on the bridge site, $\mathrm{H}_{2} \mathrm{~S}$ adsorption on the hollow site and $\mathrm{H}_{2} \mathrm{~S}$ adsorption on the top site and the subplot represents experimental power spectra of $\mathrm{Bi}_{2} \mathrm{Te}_{3}$ thin films as a function of temperature.

For higher temperature $(600 \mathrm{~K})$ the spectra shifts to $0.4 \times 10^{-3} \mathrm{~W}$ $\mathrm{m}^{-1} \mathrm{~K}^{-2}$. The top-site shows better results in comparison to other three cases. The power factor shows $2.1 \times 10^{-3} \mathrm{~W} \mathrm{~m}^{-1} \mathrm{~K}^{-2}$ at $100 \mathrm{~K}$. It increases with temperature and shows a maximum value of $12.3 \times 10^{-3} \mathrm{~W} \mathrm{~m}^{-1} \mathrm{~K}^{-2}$ around $600 \mathrm{~K}$.

Hence the monotonic increase in the power factor for topsite configuration makes it suitable for technological applications in the temperature range between $300 \mathrm{~K}$ and $500 \mathrm{~K}$. We also compare our present calculated $P^{\mathrm{av}}$ to the power factor of $\mathrm{Bi}_{2} \mathrm{Te}_{3}$ thin films deposited onto polyimide substrates as reported by Goncalves, et al. ${ }^{49}$ The films are made of $35-40 \% \mathrm{Bi}$ and $65-60 \%$ Te, which makes them almost stoichiometric. At different temperatures ranging from $423 \mathrm{~K}$ to $573 \mathrm{~K} \mathrm{Bi}$ and Te show different evaporation rates. The maximum evaporation rate found in $\mathrm{Bi}$ and $\mathrm{Te}$ at $543 \mathrm{~K}$ is $2.0 \AA \mathrm{s}^{-1}$ and $6.4 \AA \mathrm{s}^{-1}$ respectively. At this temperature $(543 \mathrm{~K})$ measurements show a greater value of power factor as compared to other substrate temperatures. This behavior demonstrates how to choose the best thin film. The top site configuration shows approximately a three times greater value in comparison to $\mathrm{Bi}_{2} \mathrm{Te}_{3}$ thin films deposited onto polyimide substrates which elucidate that the top site configuration shows a high suitability for thermoelectric devices. We must be cautious and remember that we have used the thermal conductivity of pristine graphene in calculating the power factor.

The above-mentioned physical quantities $\left(\sigma^{\mathrm{av}}, S^{\mathrm{av}}, k^{\mathrm{av}}\right.$ and $P^{\text {av }}$ ) are discussed as a function of temperature. Also we should emphasize that these quantities are very sensitive to the chemical potential therefore we have calculated and briefly discussed their relationship with chemical potential in the ESI. $\dagger$

\section{Conclusions}

We have presented the transport properties of pristine graphene, and a $\mathrm{H}_{2} \mathrm{~S}$ molecule on pristine graphene at different sites (top, bridge, and hollow). The transport properties were investigated using the Boltzmann transport theory within the full potential linear augmented plane wave (FPLAPW) method based on DFT. The exchange correlation energy was solved with the generalized gradient approximation (GGA-PBE). The average values of tensor components of electrical conductivity, thermal conductivity, Seebeck coefficient and figure of merit are discussed in detail. The investigation of the figure of merit and the average power factor suggests that the top-site configuration may be a potential candidate for thermoelectric technological applications in the temperature range 300-600 K.

\section{Acknowledgements}

The work was developed within the CENTEM project, reg. no. CZ.1.05/2.1.00/03.0088, co-funded by the ERDF as part of the Ministry of Education, Youth and Sports OP RDI programme. S.A. thanks the Council of Scientific and Industrial Research (CSIR) - National Physical Laboratory for financial support.

\section{References}

1 G. Jeffrey Snyder and E. S. Toberer, Nat. Mater., 2008, 7, 105114.

2 H. Wang, W. Chu and H. Jin, Comput. Mater. Sci., 2012, 60, 224-230.

3 K. P. Ong, D. J. Singh and P. Wu, Phys. Rev. B: Condens. Matter Mater. Phys., 2011, 83, 115110.

4 D. Parker and D. J. Singh, Phys. Rev. B: Condens. Matter Mater. Phys., 2011, 83, 233206.

5 D. Parker, M.-H. Du and D. J. Singh, Phys. Rev. B: Condens. Matter Mater. Phys., 2011, 83, 245111.

6 D. J. Singh, Phys. Rev. B: Condens. Matter Mater. Phys., 2010, 81, 195217.

7 K. C. Ong, D. J. Singh and P. Wu, Phys. Rev. Lett., 2010, 104, 176601.

8 G. B. Wilson-Short, D. J. Singh, M. Fornari and M. Suewattana, Phys. Rev. B: Condens. Matter Mater. Phys., 2007, 75, 035121.

9 D. J. Singh and D. Kasinathan, J. Electron. Mater., 2007, 36, 736.

10 D. J. Singh, Phys. Rev. B: Condens. Matter Mater. Phys., 2007, 76, 085110.

11 D. J. Singh, Oxide Thermoelectrics, MRS Symp. Proc., 2008, 1044, U02-U05.

12 D. J. Singh, Alkaline earth filled nickel skutterudite antimonide thermoelectrics, US 8,487,178, 16 July 2013.

13 J. H. Chen, M. Ishigami, C. Jang, D. R. Hines, M. S. Fuhrer and E. D. Williams, Adv. Mater., 2007, 19, 3623.

14 M. D. Stoller, S. J. Park, Y. W. Zhu, J. H. An and R. S. Ruoff, Nano Lett., 2008, 8, 3498.

15 G. Eda, G. Fanchini and M. Chhowalla, Nat. Nanotechnol., 2008, 3, 270.

16 H. Lee, J. Ihm, M. L. Cohen and S. G. Louie, Nano Lett., 2010, 10, 793.

17 F. Schedin, A. K. Geim, S. V. Morozov, E. M. Hill, P. Blake, M. I. Katsnelson and K. S. Novoselov, Nat. Mater., 2007, 6, 652. 
18 J. Seol, I. Jo, A. Moore, L. Lindsay, Z. Aitken, M. Pettes, X. Li, Z. Yao, R. Huang, D. Broido, N. Mingo, R. Ruoff and L. Shi, Science, 2010, 328, 213.

19 Y. Zuev, W. Chang and P. Kim, Phys. Rev. Lett., 2009, 102, 96807.

20 C. Palma and P. Samor, Nat. Chem., 2011, 3, 431.

21 H. Sevincli and G. Cuniberti, Phys. Rev. B: Condens. Matter Mater. Phys., 2010, 81, 113401.

22 Y. Lu, B. R. Goldsmith, N. J. Kybert and A. T. C. Johnson, Appl. Phys. Lett., 2010, 97, 083107.

23 Z. M. Ao, J. Yang, S. Li and Q. Jiang, Chem. Phys. Lett., 2008, 461, 276.

24 O. Leenaerts, B. Partoens and F. M. Peeters, Phys. Rev. B: Condens. Matter Mater. Phys., 2008, 77, 125416.

25 H. J. Yoon, D. H. Jun, J. H. Yang, Z. Zhou, S. S. Yang and M. M.-C. Cheng, Sens. Actuators, B, 2011, 157, 310.

26 J. L. Johnson, A. Behnam, S. J. Pearton and A. Ural, Adv. Mater., 2010, 22, 4877.

27 D. Li, Y. Ouyang, J. Li, Y. Sun and L. Chen, Solid State Commun., 2012, 152, 422.

28 J. Li, X. Wang, K. Liu, Y. Sun and L. Chen, Solid State Commun., 2012, 152, 386.

29 A. K. Manna and S. K. Pati, Chem. - Asian J., 2009, 4, 855.

30 W. Chen, S. Chen, D. C. Qi, X. Y. Gao and A. T. S. Wee, J. Am. Chem. Soc., 2007, 129, 10418.

31 S. Gowtham, R. H. Scheicher, R. Ahuja, R. Pandey and S. P. Karna, Phys. Rev. B: Condens. Matter Mater. Phys., 2007, 76, 033401.

32 W. H. Tao and C. H. Tsai, Sens. Actuators, B, 2002, 81, 237.

33 J. E. Castellanos Á guila, H. Hernández Cocoletzi and G. Herńandez Cocoletzi, AIP Adv., 2013, 3, 032118.

34 Y.-H. Zhang, Li-F. Han, Y.-H. Xiao, D.-Z. Jia, Z.-H. Guo and F. Li, Comput. Mater. Sci., 2013, 69, 222-228.
35 B. Amin, N. Singh, T. M. Tritt, H. N. Alshareef and U. Schwingenschlogl, Appl. Phys. Lett., 2013, 103, 031907.

36 P. Blaha, K. Schwarz, G. Madsen and D. K. J. Luitz, WIEN2K, An Augmented plane Wave Plus Local Orbitals Program for Calculating Crystal properties, Vienna University of Technology, Austria, 2001, ISBN: 3-9501031-1-2.

37 G. K. H. Madsen and D. J. Singh, Comput. Phys. Commun., 2006, 175, 67-71.

38 J. P. Perdew, K. Burke and M. Ernzerhof, Phys. Rev. Lett., 1996, 77, 3865-3868.

39 M. Zhou, Y.-H. Lu, Y.-Q. Cai, C. Zhang and Y.-P. Feng, Nanotechnology, 2011, 22, 385502.

40 H. Zhang, Q. Fu, Y. Cui, D. Tan and X. Bho, J. Phys. Chem. C, 2009, 113, 8296.

41 P. Hua, W. C. Lei, L. J. Chao, Z. Rui-Zhi, W. Hong-Chao and S. Y. Chin, Physica B, 2011, 20(4), 046103.

42 D. Wang, L. Tang, M. Q. Long and Z. G. Shuai, J. Chem. Phys., 2009, 131, 224704.

43 G. J. Snyder and E. S. Toberer, Nat. Mater., 2008, 7, 105114.

44 B. Xu, X. Li, G. Yu, J. Zhang, S. Ma, Y. Wang and L. Yi, J. Alloys Compd., 2013, 565, 22-28.

45 T. Stauber, N. M. R. Peres and F. Guina, Phys. Rev. B: Condens. Matter Mater. Phys., 2008, 76, 205423.

46 C. Kittel, Introduction to solid state physics, Wiley, 8th edn, 2005.p. 147.

47 C. J. M. Lasance, Issue, November 2006, http://www.electronicscooling.com/2006/11/the-seebeck-coefficient/.

48 D. J. Singh, Doping-dependent thermopower of PbTe from Boltzmann transport calculations, Phys. Rev. B: Condens. Matter Mater. Phys., 2010, 81, 195217.

49 L. M. Goncalves, C. Couto, P. Alpuim, A. G. Rolo, F. Völklein and J. H. Correia, Thin Solid Films, 2010, 518, 2816. 